\title{
Reflection of VLF Radio Waves From an Inhomogeneous Ionosphere. Part III. Exponential Model With Hyperbolic Transition
}

\author{
James R. Wait and Lillie C. Walters
}

Contribution from Central Radio Propagation Laboratory, National Bureau of Standards, Boulder, Colo.

(Received June 19, 1963)

\begin{abstract}
This is a continuation of two earlier papers on the subject of reflection of waves from inhomogeneous isotropic media. In this particular paper an exponential conductivity profile is perturbed in such a manner that the conductivity is increased for all heights above a certain level. A hyperbolic tangent transition is employed in order to avoid discontinuities in the conductivity versus height profile.
\end{abstract}

\section{Introduction}

In two earlier communications from the present authors [Wait and Walters 1963a and 1963b] oblique reflection of (VLF) radio waves from continuously stratified ionized media was considered. In the first of these, denoted (I), the profile of the effective conductivity was taken to be exponential in form. It was pointed out that an isotropic exponential model is a fairly good representation of the $D$ layer of the ionosphere under quiet DAYTIME conditions. In the second paper, denoted (II), an idealized perturbed exponential model was considered. The perturbation consists of a localized increase of electron density which itself has a Gaussian profile. The reflection coefficient was shown to be influenced by the vertical location of this Gaussian perturbation. In the present paper, which is part III of the series, the exponential profile model introduced in (I), is modified by allowing the electron density to be increased for all heights above a specified level. Such a modification to the quiescent ionosphere could result from ionizing radiations associated with a solar flare [Jean and Crary, 1962; Pierce, 1963; Chilton et al., 1963].

For the detailed numerical results given here, it is assumed that the earth's magnetic field may be neglected. This is justified for highly oblique incidence at VLF provided that attention is restricted to effects which result from ionization in lowest daytime ionosphere [Johler, 1962].

\section{Description of the Profile}

The notation follows that used in (I) as closely as possible. Thus, the undisturbed profile, as a function of height $z$, is defined by the conductivity parameter $1 / L(z)$ where

$$
1 / L(z)=(1 / L) \exp (\beta z),
$$

and $L$ is a constant, $\beta$ is a gradient parameter, and $z$ is the height above the reference level. Under the isotropic assumption, it is known that [Wait, 1962]

$$
L=\frac{\omega(\nu+i \omega)}{\omega_{0}^{2}},
$$

in terms of the angular frequency $\omega$, collision frequency $\nu$, and the plasma frequency $\omega_{0}$. Furthermore, at VLF, $\nu>>\omega$ which leads to

$$
L \cong \omega / \omega_{r} \text { where } \omega_{r}=\omega_{0}^{2} / \nu .
$$

In general, the conductivity parameter is proportional to $N(z) / \nu(z)$ where $N(z)$ and $\nu(z)$ are the electron density and collision frequency regarded as a function of height. The constant $\beta$, in the exponent, is a measure of the sharpness of the gradient. As in (II), it will be assumed that $\beta=0.3 \mathrm{~km}^{-1}$ typifies an undisturbed $D$-layer profile.

We shall now turn our attention to the modification of the exponential profile. As in (II), it is assumed that the collision frequency profile is unchanged whereas the ionization is to be increased by an amount $\Delta N(z)$ where

$$
\Delta N(z)=\Delta N_{0}\left[\tanh \left(\frac{z-F}{D}\right)+1\right]
$$

and $\Delta N_{0}, F$, and $D$ are constants. It is evident that $\Delta N(z)$ is equal to $\Delta N_{0}$ at $z=F$ which may be described as the "lower edge" of the modified layer. When $z$ is somewhat less than $F$ it is seen that $\Delta N(z)$ is zero whereas when $z$ is much greater than $F, \Delta N(z)$ becomes equal to $2 \Delta N_{0}$. It is clear that the vertical location of the "lower edge" is governed by the value of $F$. The rapidity of the transition from 0 to $2 \Delta N_{0}$ is controlled by the magnitude of $D$; the quantity $2 D$ could be described as the "transition thickness."

Following the discussion given in (II), the collision frequency profile is taken to be

$$
\nu(z)=\nu_{0} \exp (-\beta z / 2),
$$




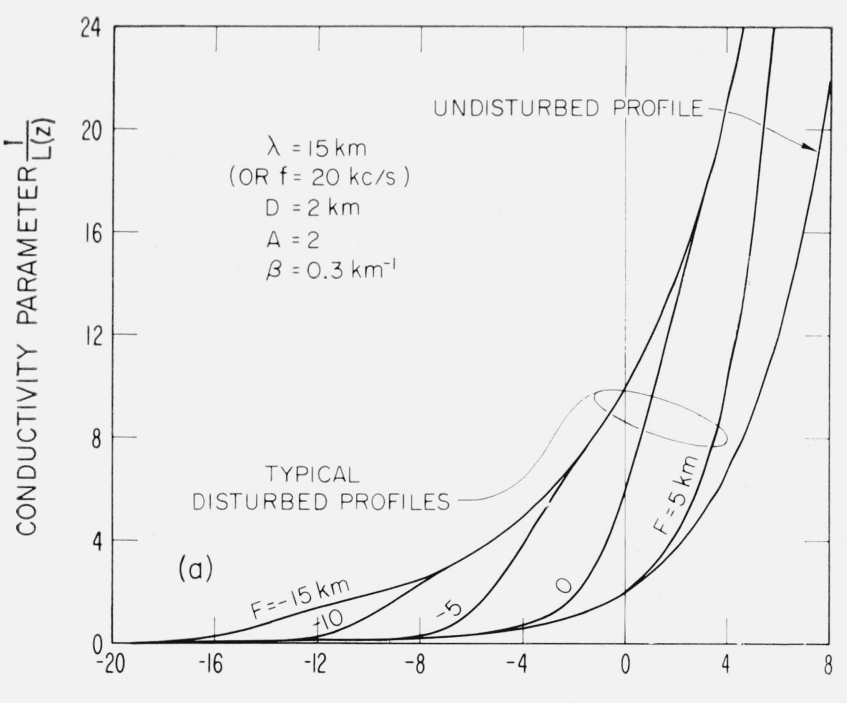

VERTICAL DISTANCE Z IN km
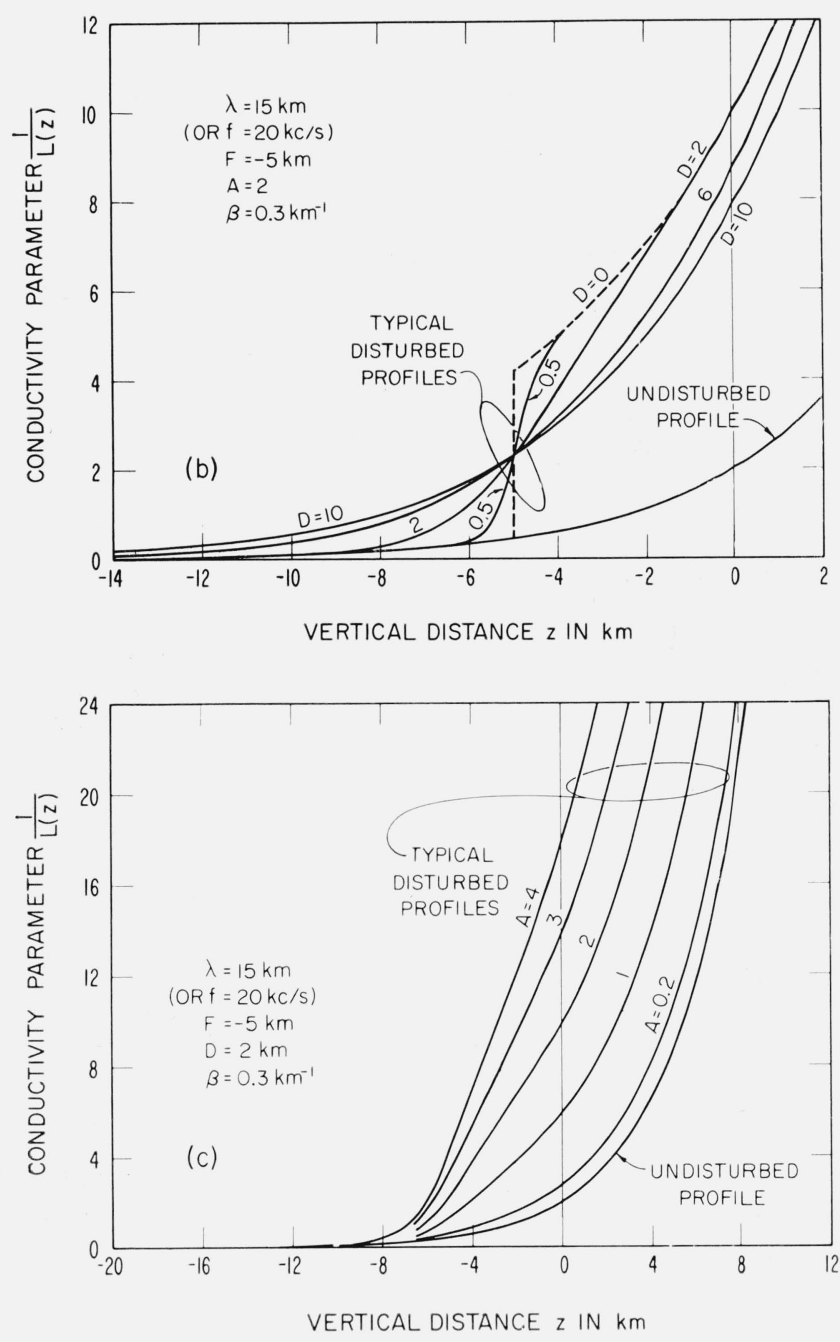

Figures 1a, 1b, 1c. The profile of the conductivity of the model illustrating the influence of the various parameters. where $\beta=0.30 \mathrm{~km}^{-1}$. The resulting conductivity perturbation has the form

$$
\frac{\Delta N(z)}{\nu(z)}=\frac{\Delta N_{0}}{\nu_{0}} \exp (\beta z / 2)\left[1+\tanh \left(\frac{z-F}{D}\right)\right] .
$$

The complete conductivity profile is now given by

$$
\begin{aligned}
\frac{1}{L(z)}=\frac{1}{L(0)}\{\exp (\beta z)+ & A \exp \left(\frac{\beta}{2} z\right) \\
& {\left.\left[1+\tanh \left(\frac{z-F}{D}\right)\right]\right\}, }
\end{aligned}
$$

where the right-hand side is proportional to the effective conductivity of the medium as a function of height above (or below) the reference level at $z=0$. The coefficient $A$ defines the strength or magnitude of the perturbation. The constant $L(0)$ is chosen to be equal to $7.5 / \lambda$ where $\lambda$ is the wavelength in kilometers.

To enable the reader to grasp the significance of the various possible forms of profiles, some typical examples are illustrated in figures $1 \mathrm{a}, 1 \mathrm{~b}$, and $1 \mathrm{c}$. In figure 1a, the vertical location of the "lower edge" of the perturbed layer is varied while other factors are kept constant. In $1 \mathrm{~b}$, the abruptness of the transition at the "lower edge" is varied for fixed values of $F$ and $A$. Finally, in 1c, the strength of the perturbed layer is varied while keeping $F$ and $D$ constant.

\section{Results of the Calculations}

The method used in the reflection coefficient calculations is the same as that used in (I) and (II), so this aspect of the subject need not be discussed. As before, the amplitude $|R|$ and the phase of $R$ for a vertically polarized wave are considered. The reflection coefficient is evaluated in the freespace region corresponding to $z \rightarrow-\infty$; however, it is to be remembered that the phase is referred to the level $z=0$.

In figure $2 \mathrm{a}$, the amplitude of the reflection coefficient is plotted as a function of $F$ for $\lambda=15 \mathrm{~km}$ (i.e., $f=20 \mathrm{kc} / \mathrm{s}$ ), $A=2, D=2 \mathrm{~km}$, and $C$ values varying from 0.05 to 0.4 . Small values of $C$ correspond to angles near grazing; these are important for long distance propagation of VLF radio waves. It is evident that when $F$ is somewhat above zero, $|R|$ assumes the value for an unperturbed exponential layer. As the "lower edge" of the perturbed layer moves down (i.e., $F$ becoming negative), the tendency is for $|R|$ to increase somewhat. As the perturbed layer is moved farther down (i.e., becoming increasingly negative), $|R|$ tends to be reduced. The behavior illustrated here is not dissimilar to the corresponding case of a Gaussian "bump," described in (II), as it moves down to low heights. In both cases, the perturbation enhances the reflection at greater heights but degrades it at lower heights.

The phase of $R$ is shown in figure $2 \mathrm{~b}$ for the same 

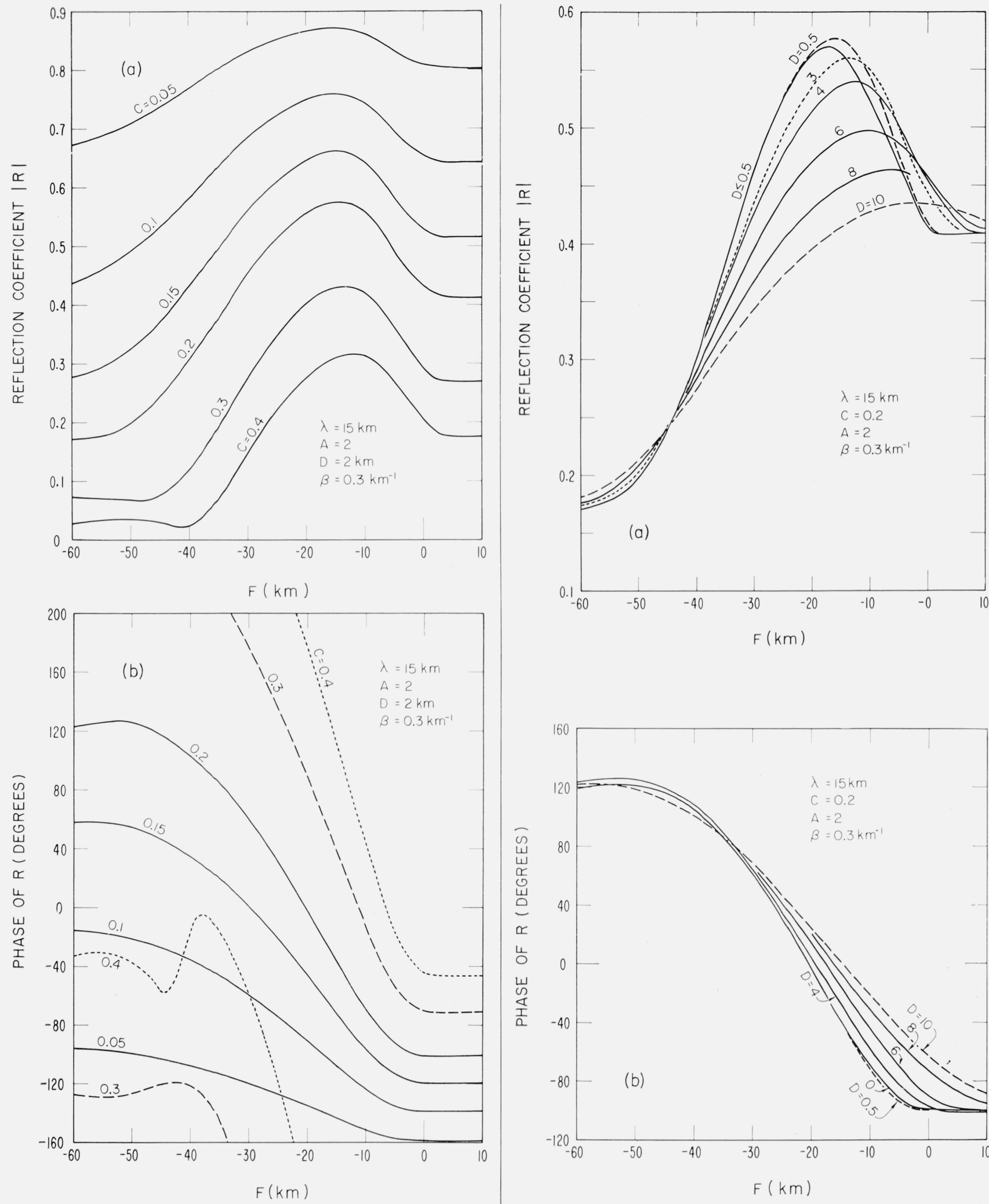

Figures 2a, 2b. Amplitude and phase of the reflection coefficient for various values of $\mathrm{C}$, the cosine of the angle of incidence. $F$ is the vertical distance, relative to the reference height, to the "lower edge" of the perturbed layer.] 

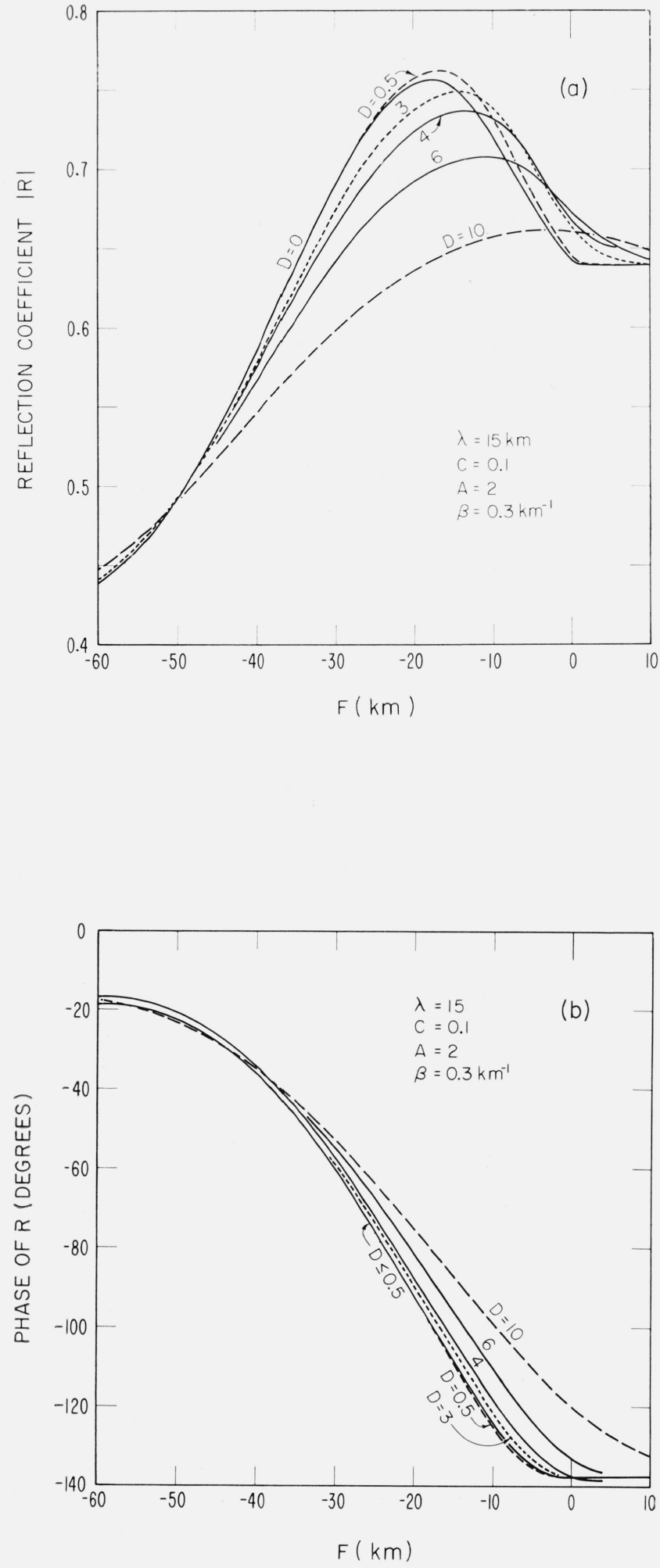

Figures 4a, 4b. Amplitude and phase of the reflection coefficient for various values of $\mathrm{D}$, the "half-thickness" of the layer transition for $\mathrm{C}=0.1$.
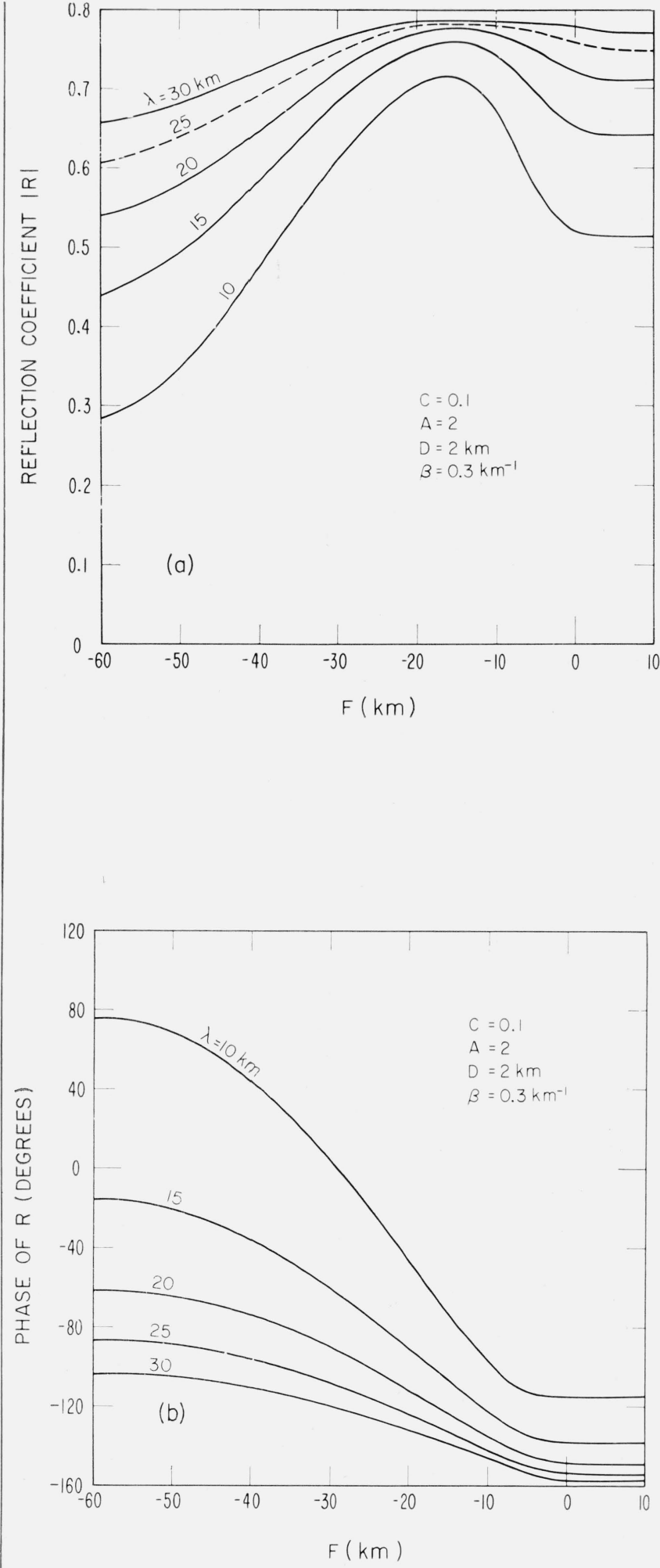

Figures 5a, 5b. Amplitude and phase of the reflection coefficient for various values of $\lambda$, the wavelength. 

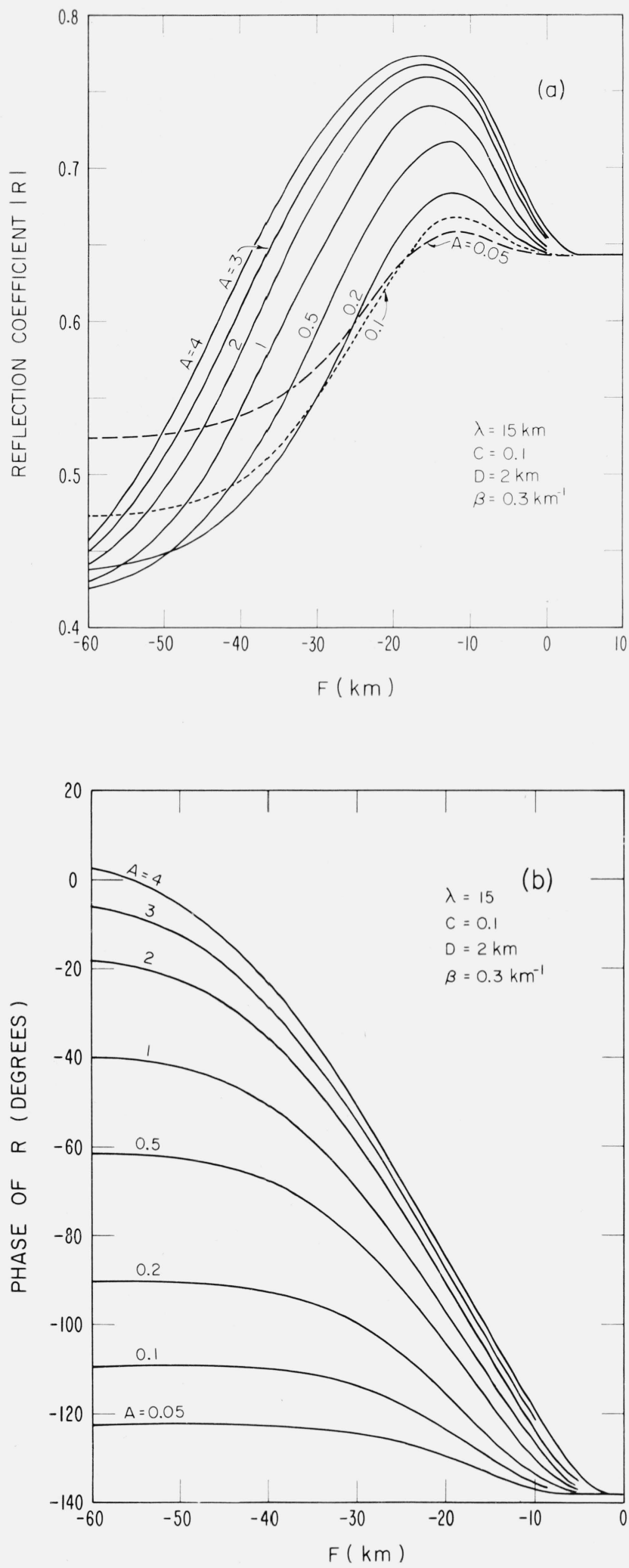

Figures 6a, 6b. Amplitude and phase of the reflection coefficient for various values of $\mathbf{A}$, the magnitude of the perturbation. conditions as in figure $2 \mathrm{a}$. It is apparent that when the "lower edge" is somewhat above $F=0$, the phase attains its undisturbed value. As $F$ becomes negative the phase increases and, for grazing angles, this increase is monotonic in nature. However, for the steeper angles of incidence there is some evidence of oscillations, which are presumably due to certain interference phenomena.

The dependence of the "transition thickness" on the reflection coefficient is shown in figures $3 \mathrm{a}$ and $3 \mathrm{~b}$ for $C=0.2$. These show, as one might expect, that a sharp transition is associated usually with an increased reflection coefficient. As the transition is stretched out, the tendency is for the maximum at $F \cong-15 \mathrm{~km}$ to disappear. It is particularly interesting to note that the phase is not overly sensitive to changes of $D$.

The influence of varying $D$ for a highly grazing situation is shown in figures $4 \mathrm{a}$ and $4 \mathrm{~b}$. These show behavior which is qualitatively the same as figures $3 \mathrm{a}$ and $3 \mathrm{~b}$.

The wavelength dependence of the reflection coefficient is shown in figures $5 \mathrm{a}$ and $5 \mathrm{~b}$ for $\lambda=10$, $15,20,25$, and $30 \mathrm{~km}$ corresponding to frequencies of $30,20,15,12$, and $10 \mathrm{kc} / \mathrm{s}$. As expected, the curves have similar shapes to each other and the tendency is for the shorter wavelengths to be associated with more pronounced changes.

Finally, in figures $6 \mathrm{a}$ and $6 \mathrm{~b}$, the influence of varying the strength $A$ of the perturbed layer is indicated. Generally, as $A$ is increased, the changes are more pronounced. It is interesting to note that at the very low heights the magnitude of the reflection coefficient is very sensitive to small changes of $A$. This behavior is similar to that found in the troposphere when considering the reflection of VHF radio waves from gradient changes of the refractive index [Wait, 1962].

\section{Concluding Remarks}

In the present series of three papers, the reflection of plane waves from an inhomogeneous isotropic medium has been considered. Attention has been confined to horizontally stratified layers whose effective conductivity varies smoothly with height. Special attention is given to vertically polarized waves at highly oblique incidence and at very low frequencies because these are important in long distance propagation.

In the first paper, the conductivity profile is taken to be exponential in form. Although this is a simple model, it is quite convenient to employ for quantitative estimates of the amplitude and phase changes as a function of gradient, angle of incidence, and frequency. The application of the results of (I) to mode theory has already been carried out [Wait, 1963].

In the second paper of this series, denoted (II), the exponential profile is allowed to have a perturbation in the form of a Gaussian curve. In the third paper (i.e., the present one), the perturbation is in the form of a hyperbolic tangent. Basically, the 
difference between these two classes of profiles is that in (II) the perturbation is localized whereas in (III) it is spread out over a range of heights. It is probable that the results in (II) are more applicable to ionization produced by the hard X-rays emitted from a space nuclear burst. On the other hand, the results in (III) would be more appropriate to the ionization produced by a solar flare.

The direct application of the results in (II) and (III) to mode theory computations and further analysis including the earth's magnetic field is reserved for future communications. In the meantime, it is hoped that the present results should add to the knowledge of wave propagation in inhomogeneous media.

We thank A. G. Jean for many useful suggestions concerning this work. The work has been supported by the Advanced Research Projects Agency, Washington, D.C., under ARPA Order No. 183-62.

\section{References}

Chilton, C. J., F. K. Steele, and R. B. Norton (1963), VLF phase observations of solar flare-produced ionization in the $D$ region of the ionosphere, J. Geophys. Res. (to be published).
Jean, A. G., and J. H. Crary (Nov. 1962), VLF phase observations on the ionospheric effects of the solar flare of Sept. 28, 1961, J. Geophys. Res. 67, 4903.

Johler, J. R. (Jul. 1962), Radio wave reflections at a continuously stratified plasma with collisions proportional to energy and arbitrary magnetic induction, Proc. London Conference on the Ionosphere (distributed by Chapman and Hall, London, 1963).

Pierce, E. T. (May, 1963), Stanford Research Institute, private communications.

Wait, J. R. (1962), Electromagnetic waves in stratified media (Pergamon Press, Oxford, New York-see chap. IV).

Wait, J. R. (Jul.-Aug. 1963), Influence of the lower atmosphere on propagation of VLF waves to great distances, J. Res. NBS 6\%D (Radio Prop.), No. 4, 375-381.

Wait, J. R., and L. C. Walters (May-June 1963a), Reflection of VLF radio waves from an inhomogeneous ionosphere. Part I. Exponentially varying isotropic model, J. Res. NBS 67D (Radio Prop.), No. 3, 361-367.

Wait, J. R., and L. C. Walters (Sept.-Oct. 1963b), Reflection of VLF radio waves from an inhomogeneous ionosphere. Part II. Perturbed exponential model, J. Res. NBS 67D (Radio Prop.), No. 5, 509-523.

\section{Additional Reference}

Belrose, J. S. (1963), Electron Density Measurements in the D Region by the Method of Partial reflection, Proceedings of NRDE Conference, Electron Density Profiles Pergamon Press, Oxford, London). In press.

(Paper 67D6-303) 